\title{
Strong iISS: combination of iISS and ISS with respect to small inputs*
}

\author{
Antoine Chaillet ${ }^{1}$, David Angeli ${ }^{2}$, Hiroshi Ito $^{3}$
}

\begin{abstract}
This paper studies the notion of Strong iISS, which imposes both integral input-to-state stability (iISS) and input-to-state stability (ISS) with respect to small inputs. This combination characterizes the robustness property, exhibited by many practical systems, that the state remains bounded as long as the magnitude of exogenous inputs is reasonably small but may diverge for stronger disturbances. We provide three Lyapunov-type sufficient conditions for Strong iISS. One is based on iISS Lyapunov functions admitting a radially nonvanishing (class $\mathcal{K}$ ) dissipation rate. However we show that it is not a necessary condition for Strong iISS. Two less conservative conditions are then provided, which are used to demonstrate that asymptotically stable bilinear systems are Strongly iISS. Finally, we discuss cascade and feedback interconnections of Strong iISS systems.
\end{abstract}

\section{INTRODUCTION}

Since its introduction by Sontag in 1989, input-to-state stability (ISS [15]) has become one of the key concepts in the analysis and control of nonlinear systems. The ISS property requires that the norm of solutions be upper-bounded by a vanishing transient term depending on the initial state, plus a term which is somewhat proportional to the magnitude of the input signal applied to the system. A strength of ISS stands in its Lyapunov characterization: any ISS system admits a storage function with a class $\mathcal{K}_{\infty}$ dissipation rate [19]. ISS provides a crucial robustness feature: for any bounded input, the resulting solutions are bounded as well (BIBS property). This property is one of the numerous robustness features guaranteed by ISS: see [17] for a survey.

Despite its indubitable success, the solutions' boundedness under arbitrary bounded input (possibly of large amplitude) makes ISS a very strong requirement in many applications. Indeed, several practical systems do provide some robustness to inputs of "reasonable" amplitude, but generate unbounded behaviours when the applied disturbance is too intense. To overcome this intrinsic limitation, the ISS property has been relaxed following two main philosophies. The first one stands in a local version of ISS (namely LISS [20]), confining both the state and the input signal to a compact neighbourhood of the origin [7]. However, LISS being actually equivalent to asymptotic stability of the system without input (0-AS), the practical relevance of LISS is conditioned by the estimate of the

*The work of A. Chaillet has received funding from the European Union Seventh Framework Programme [FP7/2007-2013] under grant agreement n257462 HYCON2 Network of excellence. The work by H. Ito is supported in part by Grant-in-Aid for Scientific Research of JSPS under grant 22560449.

${ }^{1}$ A. Chaillet is with L2S-Univ. Paris Sud 11Supélec, 3, rue Joliot-Curie, 91192 Gif-sur-Yvette, France, Antoine.Chaillet@supelec.fr. ${ }^{2}$ D. Angeli is with Dept. of Electrical and Electronic Engineering, Imperial College London, U.K. and Dip. Sistemi e Informatica, Univ. of Florence, Italy, d.angelidimperial.ac.uk. ${ }^{3} \mathrm{H}$. Ito is with the Department of Systems Design and Informatics, Kyushu Institute of Technology, 680-4 Kawazu, Iizuka 820-8502, Japan, hiroshi@ces . kyutech. ac . jp. domain of attraction and of the size of tolerated inputs [8]. The second one is known as integral input-to-state stability (iISS [16]). Instead of bounding the solution's norm by the amplitude of the applied input, it involves a term that quantifies the energy fed into the system. Its Lyapunov characterization [4] only differs from that of ISS by the fact that the dissipation rate is allowed to be just a continuous positive definite function (rather than a class $\mathcal{K}_{\infty}$ function). The fact that the dissipation rate is not necessarily unbounded, and may even vanish for large values of the state norm, explains why iISS does not guarantee the BIBS property; in other words, some iISS systems may be destabilized by inputs of arbitrarily small amplitude.

The objective of this note is to introduce and characterize an intermediate property, halfway between the robustness strengths of ISS and the generality of iISS. More precisely, we define the notion of Strong iISS as the combination of iISS, and ISS with respect to small inputs. This combination ensures that the solutions of any Strongly iISS system are globally bounded as long as the amplitude of the input signal is below a specific threshold, and, above this threshold, they inherit all properties of iISS systems.

We start by providing the necessary definitions in Section II. In Section III, we demonstrate by means of a counter-example that the intuitive characterization of Strong iISS by means of a Lyapunov function with a class $\mathcal{K}$ dissipation rate (i.e., not necessarily unbounded, but surely non vanishing for large states) is not valid in general. Alternative Lyapunov conditions for Strong iISS are provided in Section IV: in particular we show that, if ISS with respect to small inputs and forward completeness can be shown based on the same Lyapunov function, then the system is Strongly iISS. Specific results for particular classes of nonlinear systems, including bilinear systems, are also provided. Section $\mathrm{V}$ then addresses the interconnection of Strongly iISS systems: we show that, similarly to ISS but unlike iISS, Strong iISS is naturally preserved under cascade interconnection, and we recall small gain conditions for the feedback-interconnection of Strongly iISS systems. All proofs are provided in Section VI.

Notation. For a nondecreasing function $\gamma: \mathbb{R}_{\geq 0} \rightarrow \mathbb{R}_{\geq 0}$, $\gamma(\infty) \in \mathbb{R}_{\geq 0} \cup\{\infty\}$ denotes the quantity $\lim _{s \rightarrow+\infty} \gamma(s)$. A function $\alpha: \mathbb{R}_{>0} \rightarrow \mathbb{R}_{>0}$ is of class $\mathcal{P D}$ if it is continuous and positive definite. It is of class $\mathcal{K}$ if, in addition, it is increasing. It is of class $\mathcal{K}_{\infty}$ if it is of class $\mathcal{K}$ and $\alpha(\infty)=\infty . \beta: \mathbb{R}_{>0} \times \mathbb{R}_{>0} \rightarrow \mathbb{R}_{>0}$ belongs to class $\mathcal{K} \mathcal{L}$ if, given any $t \geq 0, \beta(\cdot, t) \in \mathcal{K}$ and, given any $s \geq 0$, $\beta(s, \cdot)$ is continuous, nonincreasing and tends to zero as its argument tends to infinity. Given $m \in \mathbb{N}, \mathcal{U}^{m}$ denotes the set of all measurable locally essentially bounded functions $u: \mathbb{R}_{>0} \rightarrow \mathbb{R}^{m} \cdot|\cdot|$ stands for the Euclidean norm. For a given $u \in \overline{\mathcal{U}}^{m}$ and and set $\mathcal{I} \subset \mathbb{R}_{\geq 0},\|u\|:=\operatorname{ess}_{\sup _{t>0}}|u(t)|$ and $\|u\|_{\mathcal{I}}:=\operatorname{ess~sup}_{t \in \mathcal{I}}|u(t)|$. Given a constant $\bar{R} \geq 0$, we let $\mathcal{U}_{\leq R}^{m}$ denote the set $\left\{u \in \mathcal{U}^{m}:\|u\| \leq R\right\}$. A square 
matrix is Hurwitz if all its eigenvalues have negative real parts. sat $: \mathbb{R} \rightarrow \mathbb{R}$ is the scalar saturation function defined as $\operatorname{sat}(s)=\min \{1 ;|s|\} \operatorname{sign}(s)$ for all $s \in \mathbb{R}$. Given $a \in$ $\mathbb{R} \cup\{\infty\}$, and two continuous function $f, g: \mathbb{R} \rightarrow \mathbb{R}$, we write $g(s)=\mathcal{O}(f(s))$ as $s \rightarrow a$ if $\limsup _{s \rightarrow a}|g(s) / f(s)|<\infty$. A function $V: \mathbb{R}^{n} \rightarrow \mathbb{R}_{>0}$ is called a storage function if it is continuously differentiable and satisfies $V(0)=0$ and $V(x)>0$ for all $x \neq 0$. A storage function is said to be proper if, in addition, the set $\left\{x \in \mathbb{R}^{n}: V(x) \leq c\right\}$ is compact for any fixed $c \geq 0$.

\section{DEFINITIONS}

We consider nonlinear systems with inputs:

$$
\dot{x}=f(x, u),
$$

where $x \in \mathbb{R}^{n}$ is the state, $u \in \mathcal{U}^{m}$ is the input and $f$ : $\mathbb{R}^{n} \times \mathbb{R}^{m} \rightarrow \mathbb{R}^{n}$ denotes a locally Lipschitz function satisfying $f(0,0)=0$. Given $x_{0} \in \mathbb{R}^{n}$ and an input signal $u \in \mathcal{U}^{m}$, the solution of (1) is referred to as $x\left(\cdot ; x_{0}, u\right)$ (or simply $x(\cdot)$ when the context is clear) on the time domain where it is defined.

We start by recalling the following definition of iISS, orginally introduced in [16].

Definition 1 (iISS): The system (1) is said to be Integral Input-to-State Stable if there exist a class $\mathcal{K} \mathcal{L}$ function $\beta$ and class $\mathcal{K}_{\infty}$ functions $\mu_{1}, \mu_{2}$ such that, for all $x_{0} \in \mathbb{R}^{n}$ and all $u \in \mathcal{U}^{m}$, its solution satisfies, for all $t \geq 0$,

$$
\left|x\left(t ; x_{0}, u\right)\right| \leq \beta\left(\left|x_{0}\right|, t\right)+\mu_{1}\left(\int_{0}^{t} \mu_{2}(|u(s)|) d s\right) .
$$

It is well known that iISS guarantees to (1) some robustness with respect to a class of inputs with finite energy. In particular, it was shown in [16, Proposition 6] that if the above estimate holds then, for all $u \in \mathcal{U}^{m}$ satisfying $\int_{0}^{\infty} \mu_{2}(|u(s)|) d s<\infty$, all solutions of (1) converge to the origin. On the other hand, iISS does not guarantee any robustness to non-vanishing inputs, as illustrated by the following example.

Example 1: The system

$$
\dot{x}=-\frac{x}{1+x^{2}}+u
$$

is iISS, but given any constant input $u^{\star}>0$ (even arbitrarily small), any solution starting from an initial condition $x_{0} \geq u^{\star}$ grows unbounded.

In the same way, vanishing inputs may generate unbounded solutions for iISS system; see e.g. [5], [6]. Nonetheless, many iISS systems do exhibit some robustness to vanishing inputs, or inputs with sufficiently small magnitude. This is obviously the case of all ISS systems [15], which are known to generate bounded solutions for any bounded inputs. A lot of dynamical systems can also stand exogenous inputs that are sufficiently small, but exhibit non proper behaviours for too large inputs. This is actually very common in control practice: while the plant's state remains bounded for small perturbations, too large disturbances typically generates unbounded solutions. A natural way to describe this limited robustness property would be to consider ISS only for small inputs, as in the following definition.

Definition 2 (ISS wrt small inputs): The system (1) is said to be Input-to-State Stable with respect to small inputs if there exist a constant $R>0$, a class $\mathcal{K} \mathcal{L}$ function $\beta$ and a class $\mathcal{K}_{\infty}$ function $\gamma$ such that, for all $x_{0} \in \mathbb{R}^{n}$ and all $u \in \mathcal{U}_{\leq R}^{m}$, its solution satisfies, for all $t \geq 0$,

$$
\left|x\left(t ; x_{0}, u\right)\right| \leq \beta\left(\left|x_{0}\right|, t\right)+\gamma(\|u\|) .
$$

ISS with respect to small inputs constitutes a stronger requirement than the property referred to as local ISS (LISS) in e.g. [20], [7]. Indeed, it imposes that (2) holds for small inputs, but over the whole state space $\mathbb{R}^{n}$, whereas LISS is a local property both in the state and in the input. In particular, LISS does not ensure global asymptotic stability of the origin for the unperturbed dynamics (0-GAS), while ISS with respect to small inputs does.

If the above state estimate holds for all $u \in \mathcal{U}^{m}$, then we recover the classical definition of ISS [15]. However, given a finite $R$, the above property does not provide any information on the behaviour of the system when the magnitude of the input signal $u$ overpasses $R$. In particular, the system's solutions are not even guaranteed to exist at all time when $\|u\|>R$, as illustrated by the following example.

Example 2: Consider the scalar system

$$
\dot{x}=-x+u+\xi(u) x^{2},
$$

where $\xi: \mathbb{R} \rightarrow \mathbb{R}$ denotes any locally Lipschitz function satisfying $\xi(u)=0$ for all $u \in[-1 ; 1]$ and $|\xi(u)| \geq 1$ for all $|u| \geq 2$. For instance, one can pick $\xi(u):=\operatorname{sat}(u-2)+$ sat $(u+2)$. This system can easily be shown to be ISS with respect to $u \in \mathcal{U}_{<1}^{1}$. However, for any constant $u \geq 2$, the solution satisfies $\dot{x}(t) \geq-x(t)+x(t)^{2}$, which has finite escape time for any initial state greater than 1 .

Therefore, while ISS with respect to small inputs guarantees interesting robustness properties when the system is perturbed by sufficiently small inputs, an additional requirement is needed to ensure at least the forward completeness of the system for larger inputs. The aim of this paper is to study an intermediate property, half way between the strength of ISS and the generality of iISS. We refer to this notion as Strong iISS.

Definition 3 (Strong iISS): The system (1) is said to be Strongly iISS if it is both iISS, and ISS with respect to small inputs. In other words, there exist $\beta \in \mathcal{K} \mathcal{L}$ and $\mu_{1}, \mu_{2}, \gamma \in \mathcal{K}$ such that, for all $u \in \mathcal{U}^{m}$, all $x_{0} \in \mathbb{R}^{n}$ and all $t \geq 0$, its solution satisfies the following two properties:

$$
\begin{gathered}
|x(t)| \leq \beta\left(\left|x_{0}\right|, t\right)+\mu_{1}\left(\int_{0}^{t} \mu_{2}(|u(s)|) d s\right) \\
\|u\| \leq R \Rightarrow|x(t)| \leq \beta\left(\left|x_{0}\right|, t\right)+\gamma(\|u\|) .
\end{gathered}
$$

ISS is thus a special case of Strong iISS, which itself combines ISS with respect to small inputs and iISS, which both are special cases of LISS. Figure 1 summarizes these inclusions. Example 1 illustrates the fact that iISS $\nRightarrow$ Strong iISS, while Example 2 demonstrates the fact that ISS wrt small inputs $\nRightarrow$ Strong iISS.

The rest of this paper aims at providing some insights into the Strong iISS property and to confront it with existing robustness properties.

\section{A COUNTER-EXAMPLE TO A LYAPUNOV CHARACTERIZATION}

It is well known that both ISS and iISS of (1) are equivalent to the existence of a proper storage function $V: \mathbb{R}^{n} \rightarrow \mathbb{R}_{\geq 0}$ 


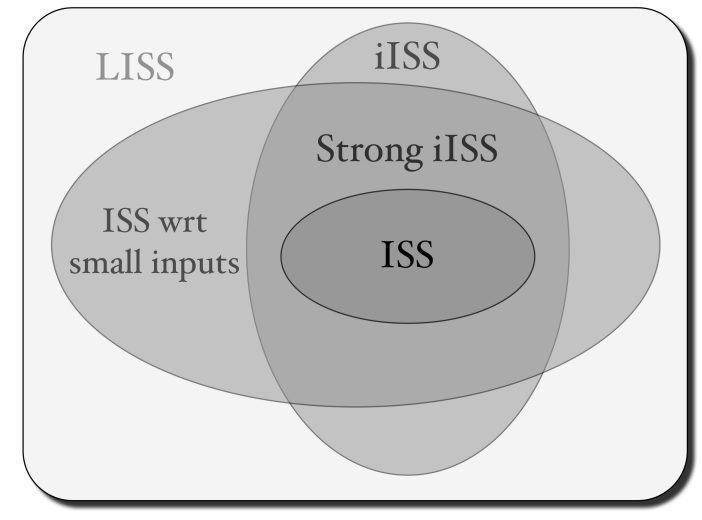

Fig. 1. Schematic hierarchy between ISS-related concepts.

and a class $\mathcal{K}_{\infty}$ function $\gamma$ satisfying for all $x \in \mathbb{R}^{n}$ and all $u \in \mathbb{R}^{m}$,

$$
\frac{\partial V}{\partial x}(x) f(x, u) \leq-\alpha(|x|)+\gamma(|u|) .
$$

The function $\alpha$ is often referred to as a dissipation rate associated to the storage function $V$. If $\alpha \in \mathcal{K}_{\infty}$, then we recover the ISS characterization, cf. [19]. If $\alpha \in \mathcal{P} \mathcal{D}$, then the above dissipation inequality is equivalent to the iISS of (1), cf. [4].

A natural conjecture is then that, when $\alpha$ is a class $\mathcal{K}$ function, this estimate is equivalent to Strong iISS. Indeed, such a dissipation rate would imply the decrease of $V$ for large values of the state if the input is of sufficiently small amplitude. While this conjecture may sound quite intuitive, it happens to be wrong. The following example shows that the necessity part of this statement is not true.

Example 3 (Strong iISS $\nRightarrow \mathcal{K}$ dissipation rate): Consider the scalar system

$$
\dot{x}=-\frac{x}{1+x^{2}}+r(|u|-1),
$$

where $r$ denotes the unit ramp ${ }^{1}$. We claim that:

a) this system is Strongly iISS;

b) given any $\alpha \in \mathcal{K}$ and $\gamma \in \mathcal{P} \mathcal{D}$, no differentiable function $V: \mathbb{R} \rightarrow \mathbb{R}_{\geq 0}$ may satisfy (3). In particular, (4) admits no storage function with a class $\mathcal{K}$ dissipation rate.

To prove Item a), notice that the total derivative of the function $V_{1}(x):=\frac{1}{2} \ln \left(1+x^{2}\right)$ along the solutions of (4) yields

$$
\dot{V}_{1} \leq-\frac{x^{2}}{\left(1+x^{2}\right)^{2}}+r(|u|-1) \leq-\frac{x^{2}}{\left(1+x^{2}\right)^{2}}+|u|,
$$

which establishes iISS by noticing that $s \mapsto s^{2} /\left(1+s^{2}\right)^{2}$ is a $\mathcal{P D}$ function. In addition, for all $|u| \leq 1$, the derivative of $V_{2}(x)=x^{4} / 4$ yields

$$
\dot{V}_{2} \leq-\frac{x^{4}}{1+x^{2}} \leq-\frac{x^{4}}{1+x^{2}}+|u|
$$

which establishes ISS with respect to inputs in $\mathcal{U}_{\leq 1}^{1}$. Item a) follows.

$$
{ }^{1} r(s)=0 \text { for all } s \leq 0 \text {, and } r(s)=s \text { for all } s>0 .
$$

Now, consider any differentiable function $V: \mathbb{R} \rightarrow \mathbb{R}_{\geq 0}$. Then it holds that

$$
\dot{V}=-\frac{\partial V}{\partial x}(x) \frac{x}{1+x^{2}}+\frac{\partial V}{\partial x}(x) r(|u|-1) .
$$

Therefore, the dissipation inequality (3) may only be satisfied with $\alpha \in \mathcal{K}$ if the term $\frac{\partial V}{\partial x}(x) \frac{x}{1+x^{2}}$ does not vanish for large state values (as it is the only negative term in (5)). This imposes in particular that

$$
\liminf _{x \rightarrow \infty} \frac{1}{x} \frac{\partial V}{\partial x}(x) \geq c
$$

for some constant $c>0$. Considering $u=2$, it then follows from (5) that

$$
\liminf _{x \rightarrow+\infty} \dot{V} \geq \lim _{x \rightarrow+\infty} \operatorname{cxr}(1)=+\infty,
$$

whereas the right-hand side of (3) imposes $\dot{V} \leq \gamma(2)$ for all $x \in \mathbb{R}$. This contradiction establishes Item $\mathrm{b}$ ).

\section{SUFFICIENT CONDITIONS FOR STRONG IISS}

\section{A. Class $\mathcal{K}$ dissipation rate}

While the necessity part of this conjecture is contradicted by Example 3, the existence of a storage function with a class $\mathcal{K}$ dissipation rate does guarantee the Strong iISS property. We formally state this sufficient condition in the following result.

Theorem 1: Assume that there exists a proper storage function $V: \mathbb{R}^{n} \rightarrow \mathbb{R}_{\geq 0}$ satisfying, for all $x \in \mathbb{R}^{n}$ and all $u \in \mathbb{R}^{m}$,

$$
\frac{\partial V}{\partial x}(x) f(x, u) \leq-\alpha(|x|)+\gamma(|u|),
$$

where $\alpha \in \mathcal{K}$ and $\gamma \in \mathcal{P} \mathcal{D}$. Then the system (1) is Strongly iISS.

The dissipation inequality (7) clearly implies iISS [4]. In addition, for sufficiently small inputs, a too large increase of the state norm makes the total derivative of $V$ negative, thus inducing a bounded input-bounded state property, which ensures ISS with respect to small inputs. The full proof is provided in Section VI-A.

Note that Example 3 disallows the converse of Theorem 1. In other words, the class of iISS systems with $\mathcal{K}$ dissipation rate is a strict subset of the class of Strongly iISS systems. Thus, the next sections are devoted to the development of less conservative sufficient conditions for Strong iISS.

\section{B. A characterization conjecture}

The following statement constitutes an alternative sufficient condition for Strong iISS. Its proof is provided in Section VI-B.

Theorem 2: Assume that there exists a proper storage function $V: \mathbb{R}^{n} \rightarrow \mathbb{R}_{\geq 0}, \rho \in \mathcal{P D}, \eta \in \mathcal{K}_{\infty}$ and $\kappa \in \mathcal{K}$ such that, for all $x \in \mathbb{R}^{n}$ and all $u \in \mathbb{R}^{m}$,

$$
\frac{\partial V}{\partial x} f(x, u) \leq-\rho(|x|)+\eta(\max \{0 ;|u|-\kappa(|x|)\}) .
$$

Then the system (1) is Strongly iISS.

It is worth stressing that (8) can be equivalently written in the following decomposed form:

$$
\begin{array}{r}
\dot{V} \leq-\rho(|x|)+\eta(|u|) \\
|u| \leq \kappa(|x|) \Rightarrow \dot{V} \leq-\rho(|x|),
\end{array}
$$


where $\dot{V}$ denotes $\frac{\partial V}{\partial x}(x) f(x, u)$. Note that, in contrast to Theorem $1, \rho$ is not required to be of class $\mathcal{K}$. The first of these two dissipation inequalities corresponds to the iISS characterization [4], while the second one ensures ISS with respect to small inputs (see the proof in Section VI-B for details).

These combined properties can easily be checked on Example 3 with the function $V(x)=\frac{1}{2} \ln \left(1+x^{2}\right)$ by picking $\kappa(s)=$ $\operatorname{sat}(s)$ for all $s \geq 0$. On the other hand, in view of (9)-(10), any function $V$ fulfilling the assumptions of Theorem 1 also satisfies the requirements of Theorem 2. These observations show that Theorem 2 is strictly less conservative than Theorem 1 .

Although we have not yet succeeded in establishing this fact, we conjecture that the dissipation inequality (8) is actually a necessary condition for Strong iISS.

Conjecture 1: The converse of Theorem 2 holds.

\section{Link with forward completeness}

As illustrated by Example 2, unlike ISS with respect to small inputs, Strong iISS guarantees that the system is forward complete even for large inputs. The following result states that if both ISS with respect to small inputs and forward completeness can be established through the same Lyapunov function, then the system is Strongly iISS.

Theorem 3: Assume that there exist a proper storage function $V: \mathbb{R}^{n} \rightarrow \mathbb{R}_{\geq 0}$, some functions $\alpha, \gamma \in \mathcal{K}$, two constants $R_{x}, R_{u} \geq 0$ and three continuous nondecreasing functions $\eta, \nu_{1}, \nu_{2}: \mathbb{R}_{\geq 0} \rightarrow \mathbb{R}_{\geq 0}$ such that, for all $x \in \mathbb{R}^{n}$ and all $u \in \mathbb{R}^{m}$,

$$
\begin{gathered}
|u| \leq R_{u} \Rightarrow \frac{\partial V}{\partial x} f(x, u) \leq-\alpha(|x|)+\gamma(|u|) \\
|x| \geq R_{x} \Rightarrow \frac{\partial V}{\partial x} f(x, u) \leq \nu_{1}(|u|) \eta(V)+\nu_{2}(|u|) .
\end{gathered}
$$

Assume also that

$$
\eta(s)=\mathcal{O}(s), \quad \text { as } s \rightarrow+\infty .
$$

Then the system (1) is Strongly iISS.

Note that (11) immediately implies that (1) is ISS with respect to small inputs [19]; the contribution of the above result is mostly to establish iISS. Its proof is provided in Section VIC.

In view of [3], the condition (12) together with the sublinearity requirement (13) constitutes a characterization of the forward completeness of (1). Consequently, Theorem 3 basically states that if the forward completeness and the ISS with respect to small inputs can be established through the study of the same Lyapunov function, then the system is also iISS (thus Strongly iISS).

An extension of the counter-example [4, Section V] shows that, in general, ISS with respect to small inputs and forward completeness are not enough to guarantee iISS; the use of a common Lyapunov function for the two properties is therefore crucial for the result.

The assumptions of Theorem 1 clearly guarantee those of Theorem 3. In addition, the Strong iISS of the system in Example 3 can be checked by means of Theorem 3 with the function $V(x)=\frac{1}{2} \ln \left(1+x^{2}\right)$. This shows that Theorem 3 is strictly less conservative than Theorem 1.
Theorem 3 allows to recover and extend a result originally presented in [16].

Corollary 1: Assume that there exist a proper storage function $V: \mathbb{R}^{n} \rightarrow \mathbb{R}_{\geq 0}$, class $\mathcal{K}_{\infty}$ functions $\lambda$ and $\gamma$, and a positive constant $q$ such that, for all $x \in \mathbb{R}^{n}$ and all $u \in \mathbb{R}^{m}$,

$$
\frac{\partial V}{\partial x}(x) f(x, u) \leq-(q-\lambda(|u|)) V(x)+\gamma(|u|) .
$$

Then the system (1) is Strongly iISS.

This dissipation inequality was used in [16, Theorem 2] to establish iISS. Corollary 1 states that Strong iISS actually holds under the same condition. Its proof is omitted due to space constraints.

\section{Systems with specific structures}

We now show that Hurwitz linear systems perturbed by sector-bounded disturbances are Strongly iISS. We summarize this fact though the following proposition, whose proof is omitted.

Proposition 1: Given $A \in \mathbb{R}^{n \times n}$, consider the system

$$
\dot{x}=A x+\varphi(x, u),
$$

where $\varphi: \mathbb{R}^{n} \times \mathbb{R}^{m} \rightarrow \mathbb{R}^{n}$ is a locally Lipschitz function satisfying, for all $x \in \mathbb{R}^{n}$ and all $u \in \mathbb{R}^{m}$,

$$
|\varphi(x, u)| \leq\left(c_{1}+c_{2}|x|\right) g(|u|),
$$

for some constants $c_{1}, c_{2} \geq 0$ and $g \in \mathcal{P} \mathcal{D}$. Then (14) is Strongly iISS if and only if $\mathrm{A}$ is Hurwitz.

We stress that, when the constant $c_{2}$ in (15) is zero, then the system (14) is actually ISS. On the other hand, for a perturbation that violates (15) with $c_{2}=0$, it can be shown that (14) is no longer ISS.

Remark 1 (Strong iISS of bilinear systems): Systems of the form (14) satisfying (15) include, in particular, the class of bilinear systems:

$$
\dot{x}=\left(A+\sum_{i=1}^{m} u_{i} A_{i}\right) x+B u,
$$

where $A \in \mathbb{R}^{n \times n}, A_{i} \in \mathbb{R}^{n \times n}$ for all $i \in\{1, \ldots, m\}$ and $u=\left(u_{1}, \ldots, u_{m}\right)^{T}$. A necessary and sufficient condition (namely, $A$ Hurwitz) for the iISS of such systems was already established in [16, Theorem 5]. The above proposition shows that ISS with respect to small inputs (hence Strong iISS) also holds under the same condition.

\section{E. Exploiting zero-output dissipativity}

Exploiting the notion of zero-output smooth dissipativity, we can derive an alternative growth order condition on the supply functions for Strong iISS. This notion, introduced in [4], imposes the existence of a proper storage function $W: \mathbb{R}^{n} \rightarrow$ $\mathbb{R}_{\geq 0}$ satisfying, for all $x \in \mathbb{R}^{n}$ and all $u \in \mathbb{R}^{m}$,

$$
\frac{\partial W}{\partial x}(x) f(x, u) \leq \sigma(|u|)
$$

where $\sigma$ denotes a class $\mathcal{K}$ function. The following result is a consequence of Theorem 1 that exploits this property.

Proposition 2: Assume that (1) is zero-output smooth dissipative and that there exist a differentiable function $V_{0}: \mathbb{R}^{n} \rightarrow$ 
$\mathbb{R}_{\geq 0}$ and $\kappa_{0}, \alpha_{0}, \eta_{0}, \gamma_{0} \in \mathcal{K}$ such that, for all $x \in \mathbb{R}^{n}$ and all $u \in \mathbb{R}^{m}$,

$$
\begin{gathered}
V_{0}(x) \geq \kappa_{0}(|x|) \\
\frac{\partial V_{0}}{\partial x} f(x, u) \leq-\alpha_{0}\left(V_{0}\right)+\eta_{0}\left(V_{0}\right) \gamma_{0}(|u|),
\end{gathered}
$$

with $\eta_{0}$ and $\alpha_{0}$ satisfying

$$
\eta_{0}(s)=\mathcal{O}\left(\alpha_{0}(s)\right) \quad \text { as } s \rightarrow \sup _{x \in \mathbb{R}^{n}} V_{0}(x) .
$$

Then there exists a proper storage function $V: \mathbb{R}^{n} \rightarrow \mathbb{R}_{\geq 0}$ satisfying (3) with a class $\mathcal{K}$ dissipation rate $\alpha$ and $\gamma \in \mathcal{P} \mathcal{D}$. In particular, (1) is Strongly iISS.

The proof of this result is omitted due to space constraints. Is is worth noting that (18) is actually a direct consequence of 0 -GAS of (1), cf. [4, Lemma IV.10]. The main requirement of the above definition therefore stands on the growth constraint (19). Note that, due to the continuity of $\eta_{0}$ and $\alpha_{0}$ on $\mathbb{R}_{\geq 0}$, this condition is trivial if $V_{0}$ is bounded.

Note indeed that, in this statement, the function $V_{0}$ is neither required to be zero at zero, nor to be radially unbounded. Condition (17) only imposes that $V_{0}$ is positive for all $x \in$ $\mathbb{R}^{n} \backslash\{0\}$ and that it does not vanish for large values of the state norm. If, however, $V_{0}$ is a proper storage function, then the assumption of zero-output smooth dissipativity can be replaced by a growth condition on $\eta_{0}$, as summarized by the following statement whose proof is omitted.

Corollary 2: Assume that (18) and (19) hold for all $x \in \mathbb{R}^{n}$ and all $u \in \mathbb{R}^{m}$, with a proper storage function $V_{0}: \mathbb{R}^{n} \rightarrow$ $\mathbb{R}_{\geq 0}$ and class $\mathcal{K}$ functions $\kappa_{0}, \alpha_{0}, \eta_{0}$ and $\gamma_{0}$. Assume also that

$$
\int_{0}^{+\infty} \frac{d s}{1+\eta_{0}(s)}=+\infty .
$$

Then there exists a proper storage function $V: \mathbb{R}^{n} \rightarrow \mathbb{R}_{\geq 0}$ satisfying (3) with a class $\mathcal{K}$ dissipation rate $\alpha$ and $\gamma \in \mathcal{P} \mathcal{D}$. In particular, (1) is Strongly iISS.

\section{INTERCONNECTING STRONGLY IISS SYSTEMS}

\section{A. Cascade interconnection}

As already stressed, Strong iISS offers an interesting tradeoff between ISS and iISS. It is well known that, although cascade interconnections naturally preserves the ISS property [18], this is not the case for iISS in general, cf. e.g. [5], [6], [10]. Those references underline the need of additional assumptions for a cascade of iISS to be itself iISS, either on the supply rates of the associated Lyapunov functions, or on the decay rate of the trajectories. The result below shows that no such additional requirement is needed for Strong iISS.

Theorem 4: Let $f_{1}: \mathbb{R}^{n_{1}} \times \mathbb{R}^{n_{2}} \rightarrow \mathbb{R}^{n_{1}}$ and $f_{2}: \mathbb{R}^{n_{2}} \times$ $\mathbb{R}^{m_{2}} \rightarrow \mathbb{R}^{n_{2}}$ denote two locally Lipschitz functions and assume that the systems $\dot{x}_{1}=f_{1}\left(x_{1}, u_{1}\right)$ and $\dot{x}_{2}=f_{2}\left(x_{2}, u_{2}\right)$ are Strongly iISS with respect to $u_{1}$ and $u_{2}$ respectively. Then the cascade

$$
\begin{aligned}
& \dot{x}_{1}=f_{1}\left(x_{1}, x_{2}\right) \\
& \dot{x}_{2}=f_{2}\left(x_{2}, u_{2}\right) .
\end{aligned}
$$

is Strongly iISS with respect to $u_{2}$.

A similar result was established in [10] by relying on iISS Lyapunov functions with class $\mathcal{K}$ dissipation rates. In view of
Example 3, Theorem 4 is thus strictly less conservative than that result.

The proof of this result cannot be included due to space constraints. It is made of two steps: 1) showing the uniform asymptotic gain property (UAG) for sufficiently small inputs, which is shown in [20] to be equivalent to ISS with respect to small inputs; 2) establishing the bounded energy frequently bounded state (BEFBS) property which, combined to 0-GAS, is equivalent to iISS [2].

If (21b) is only iISS (rather that Strongly iISS), then the cascade (21) can be shown to be itself iISS.

Corollary 3: Assume that the system $\dot{x}_{1}=f_{1}\left(x_{1}, u_{1}\right)$ is Strongly iISS with respect to $u_{1}$ and that $\dot{x}_{2}=f_{2}\left(x_{2}, u_{2}\right)$ is iISS with respect to $u_{2}$. Then the cascade (21) is iISS with respect to $u_{2}$.

Since Strong iISS implies 0-GAS by definition, the following statement is a direct consequence of Theorem 4 for the case when no exogenous inputs affect the system.

Corollary 4: Assume that $\dot{x}_{1}=f_{1}\left(x_{1}, u_{1}\right)$ is Strongly iISS and that $\dot{x}_{2}=f_{2}\left(x_{2}\right)$ is globally asymptotically stable (GAS). Then the cascade

$$
\begin{aligned}
& \dot{x}_{1}=f_{1}\left(x_{1}, x_{2}\right) \\
& \dot{x}_{2}=f_{2}\left(x_{2}\right) .
\end{aligned}
$$

is GAS.

\section{B. Feedback interconnection}

The feedback interconnection of not necessarily ISS systems has already been addressed in the literature, and Lyapunovbased conditions for iISS have been provided in the literature. See for instance [1] for the case of a feedback interconnection without exogenous input and [12], [9], [11] for the case of systems with inputs. See also [13] for an extension to more than two interconnected subsystems. These references clearly establish the necessity for the storage functions associated to each subsystem to have a class $\mathcal{K}$ dissipation rate (unless the supply function of each subsystem is radially vanishing). In view of Theorem 1, this means that in practice Strong iISS of each individual subsystem is a necessary requirement for stability analysis through a small-gain argument based on the subsystems' dissipation inequalities.

\section{PROOFS}

\section{A. Proof of Theorem 1}

Since $\alpha \in \mathcal{K} \subset \mathcal{P D}$, iISS of (1) directly follows from the classical iISS characterization [4]. In addition, consider any $R>0$ such that $\gamma(R) \leq \frac{1}{4} \alpha(\infty)$. The existence of such a $R$ is ensured by the fact that $\alpha \in \mathcal{K}$ and $\gamma \in \mathcal{P D}$. Note that the function $\alpha$ is then invertible over $[0 ; 2 \gamma(R)]$ and, given any $|u| \leq R$,

$$
|x| \geq \alpha^{-1} \circ 2 \gamma(|u|) \Rightarrow \frac{\partial V}{\partial x} f(x, u) \leq-\frac{\alpha(|x|)}{2} .
$$

Invoking classical results such as [14, Theorem 4.18], it follows that the solutions of (1) are bounded for all inputs in $\mathcal{U}_{<R}^{m}$ and that there exists a class $\mathcal{K}_{\infty}$ function $\sigma$ such that, for all $x_{0} \in$ $\mathbb{R}^{n}$,

$$
\limsup _{t \rightarrow \infty}\left|x\left(t ; x_{0}, u\right)\right| \leq \sigma(\|u\|), \quad \forall u \in \mathcal{U}_{\leq R}^{m}
$$


This corresponds to the asymptotic gain property [20]. Noticing that the system is also $0-\mathrm{GAS}$, we conclude from [20, Theorem 1] that the system is ISS with respect to all $u \in \mathcal{U}_{\leq R}^{m}$.

\section{B. Proof of Theorem 2}

The dissipation inequality (9) is known to establish iISS [4], so there is only ISS with respect to small inputs left to prove. To that end, let $R$ denote any positive constant such that $R<$ $\kappa(\infty)$. Then, for all $|u| \leq R$, it holds that

$$
|x| \geq \kappa^{-1}(|u|) \quad \Rightarrow \quad \frac{\partial V}{\partial x}(x) f(x, u) \leq-\rho(|x|) .
$$

Reasoning as in the proof of Theorem 1, we conclude that (1) is ISS with respect to inputs $u \in \mathcal{U}_{\leq R}^{m}$.

\section{Proof of Theorem 3}

Assuming that (11) holds for some $\alpha, \gamma \in \mathcal{K}$ and $R_{u} \geq 0$, we have in particular that, for all $x \in \mathbb{R}^{n}$,

$$
\frac{\partial V}{\partial x}(x) f(x, 0) \leq-\alpha(|x|) .
$$

Following the same reasoning as in the proof of $[4$, Lemma IV.10], this ensures the existence of $\lambda_{1}: \mathbb{R}_{\geq 0} \rightarrow \mathbb{R}_{\geq 0}$ continuous and increasing and $\lambda_{2} \in \mathcal{K}$ such that, for all $x \in \overline{\mathbb{R}}^{n}$ and all $u \in \mathbb{R}^{m}$,

$$
\frac{\partial V}{\partial x}(x) f(x, u) \leq-\alpha(|x|)+\lambda_{1}(|x|) \lambda_{2}(|u|) .
$$

In particular

$$
|x| \leq R_{x} \Rightarrow \frac{\partial V}{\partial x} f(x, u) \leq \lambda_{1}\left(R_{x}\right) \lambda_{2}(|u|) .
$$

Now, consider the function $W(x):=\ln (1+V(x))$ for all $x \in$ $\mathbb{R}^{n}$. Noticing that the continuity of $\eta$ together with (13) ensures that the existence of $\bar{\eta}>0$ such that $\eta(V(x)) \leq \bar{\eta} V(x)$ for all $|x| \geq R_{x}$, Equation (12) ensures that, for all $|x| \geq R_{x}$,

$$
\begin{aligned}
\frac{\partial W}{\partial x} f(x, u) & \leq \frac{\eta(V)}{1+V} \nu_{1}(|u|)+\frac{\nu_{2}(|u|)}{1+V} \\
& \leq \bar{\eta} \nu_{1}(|u|)+\nu_{2}(|u|) .
\end{aligned}
$$

In the same way, (23) yields

$$
|x| \leq R_{x} \Rightarrow \frac{\partial W}{\partial x} f(x, u) \leq \lambda_{1}\left(R_{x}\right) \lambda_{2}(|u|) .
$$

It follows from (24) and (25) that

$$
\frac{\partial W}{\partial x} f(x, u) \leq \nu(|u|), \quad \forall x \in \mathbb{R}^{n}, u \in \mathbb{R}^{m},
$$

where $\nu: \mathbb{R}_{\geq 0} \rightarrow \mathbb{R}_{\geq 0}$ is the nondecreasing continuous function defined as $\nu(\cdot):=\bar{\eta} \nu_{1}(\cdot)+\nu_{2}(\cdot)+\lambda_{1}\left(R_{x}\right) \lambda_{2}(\cdot)$. Since $\nu$ is not necessarily a class $\mathcal{K}$ function, zero-output dissipativity does not follow yet. Nonetheless, (11) guarantees that

$$
|u| \leq R_{u} \Rightarrow \frac{\partial W}{\partial x} f(x, u) \leq \gamma(|u|) .
$$

Therefore, defining

$$
\tilde{\gamma}(s):=\left\{\begin{array}{cl}
\gamma(s) & \text { if } \quad s \leq R_{u} / 2 \\
a s+b & \text { if } \quad s \in\left(R_{u} / 2 ; R_{u}\right] \\
\nu(s)+2 \gamma(s) & \text { if } \quad s>R_{u},
\end{array}\right.
$$

where $a$ and $b$ are conveniently chosen to ensure continuity, $\tilde{\gamma}$ is a class $\mathcal{K}$ function and it holds that $\frac{\partial W}{\partial x} f(x, u) \leq \tilde{\gamma}(|u|)$ for all $x \in \mathbb{R}^{n}$ and all $u \in \mathbb{R}^{m}$. It follows that (1) is zerooutput dissipative. Consequently, recalling that it is 0-GAS in view of (11) and invoking [4, Theorem 1], it is also iISS. The conclusion follows by noticing that ISS with respect to small inputs trivially results from (11).

\section{REFERENCES}

[1] D. Angeli and A. Astolfi. A tight small-gain theorem for not necessarily ISS systems. Systems \& Control Letters, 56(1):87-91, 2007.

[2] D. Angeli, B. Ingalls, E.D. Sontag, and Y. Wang. Separation principles for input-output and integral-input-to-state stability. SIAM J. on Contr. and Opt., 43(1):256-276, 2004.

[3] D. Angeli and E.D. Sontag. Forward completeness, unboundedness observability, and their Lyapunov characterizations. Syst. \& Contr. Letters, 38:209-217, 1999.

[4] D. Angeli, E.D. Sontag, and Y. Wang. A characterization of integral input to state stability. IEEE Trans. on Automat. Contr., 45:1082-1097, 2000.

[5] M. Arcak, D. Angeli, and E.D. Sontag. A unifying integral ISS framework for stability of nonlinear cascades. SIAM J. on Contr. and Opt., 40:888-1904, 2002.

[6] A. Chaillet and D. Angeli. Integral Input-to-State Stable systems in cascade. Systems \& Control Letters, 57(7):519-527, 2008.

[7] S.N. Dashkovskiy, M. Görges, and L. Naujok. Local Input-to-State Stability of production networks. In Proc. of the Second International Conference LDIC, pages 1-10, August 2009.

[8] S.N. Dashkovskiy, B.S. Rueffer, and F.R. Wirth. Numerical verification of local input-to-state stability for large networks. In 46th IEEE Conference on Decision and Control, pages 4471-4476, 2007.

[9] H. Ito. State-dependent scaling problems and stability of interconnected iISS and ISS systems. IEEE Trans. on Automatic Control, 51(10):1626-1643, 2006

[10] H. Ito. A Lyapunov approach to cascade interconnection of Integral Input-to-State Stable systems. IEEE Trans. on Automat. Contr. 55(3):702-708, 2010

[11] H. Ito, R.A. Freeman, and A. Chaillet. Revisiting the iISS smallgain theorem through transient plus ISS small-gain regulation. Asian Journal of Control, 15(3):1-9, 2013.

[12] H. Ito and Z.P. Jiang. Necessary and sufficient small gain conditions for integral Input-to-State Stable systems: A Lyapunov perspective. IEEE Trans. on Automat. Contr., 54:2389-2404, 2009.

[13] H. Ito, Z.P. Jiang, S.N. Dashkovskiy, and B.S. Rueffer. A smallgain theorem and construction of sum-type Lyapunov functions for networks of iISS systems. In American Control Conference (ACC), pages 1971-1977, 2011.

[14] H. Khalil. Nonlinear systems. Prentice Hall, 3rd ed., New York, 2001.

[15] E.D. Sontag. Smooth stabilization implies coprime factorization. IEEE Trans. on Automat. Contr., 34(4):435-443, 1989.

[16] E.D. Sontag. Comments on integral variants of ISS. Syst. \& Contr. Letters, 34:93-100, 1998.

[17] E.D. Sontag. Input to state stability: Basic concepts and results, chapter in Nonlinear and Optimal Control Theory, pages 163-220. Lecture Notes in Mathematics. Springer-Verlag, Berlin, 2006. P. Nistri and G. Stefani eds.

[18] E.D. Sontag and A.R. Teel. Changing supply functions in Input-toState Stable systems. IEEE Trans. on Automat. Contr., 40(8):1476$1478,1995$.

[19] E.D. Sontag and Y. Wang. On characterizations of the Input-to-State Stability property. Syst. \& Contr. Letters, 24:351-359, 1995.

[20] E.D. Sontag and Y. Wang. New characterizations of Input-to-State Stability. IEEE Trans. on Automat. Contr., 41:1283-1294, 1996. 